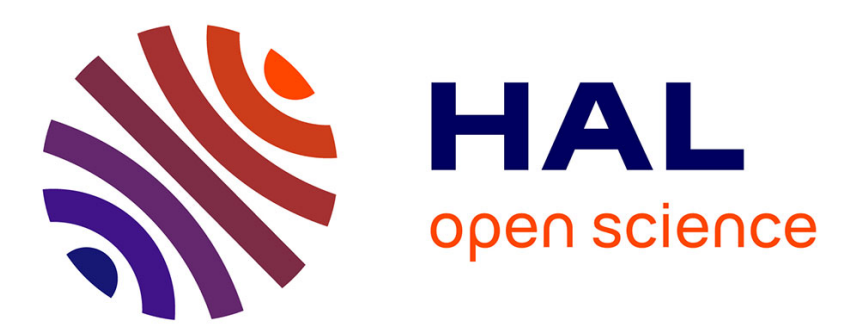

\title{
An explicit 2D finite element time domain scheme for electromagnetic wave propagation
}

\author{
Alain Nicolas, Laurent Nicolas, Christian Vollaire
}

\section{To cite this version:}

Alain Nicolas, Laurent Nicolas, Christian Vollaire. An explicit 2D finite element time domain scheme for electromagnetic wave propagation. IEEE Transactions on Magnetics, 1999, 35 (3 Part 1), pp.15381541. hal-00141541

\author{
HAL Id: hal-00141541 \\ https://hal.science/hal-00141541
}

Submitted on 18 Apr 2007

HAL is a multi-disciplinary open access archive for the deposit and dissemination of scientific research documents, whether they are published or not. The documents may come from teaching and research institutions in France or abroad, or from public or private research centers.
L'archive ouverte pluridisciplinaire HAL, est destinée au dépôt et à la diffusion de documents scientifiques de niveau recherche, publiés ou non, émanant des établissements d'enseignement et de recherche français ou étrangers, des laboratoires publics ou privés. 


\title{
An Explicit 2D Finite Element Time Domain Scheme for Electromagnetic Wave Propagation
}

\author{
A. Nicolas, L. Nicolas, C. Vollaire \\ CEGELY - UPRESA CNRS 5005 - Ecole Centrale de Lyon \\ BP163 - 69131 Ecully cedex - France
}

\begin{abstract}
An explicit 2D FETD formulation for electromagnetic wave propagation is presented. The space dirscretization is obtained using finite element triangles. The domain is truncated using an Higdon absorbing boundary condition. The time discretization is performed using a four level scheme. Mass lumping technique makes it explicit. Several numerical parameters are studied, such as the mesh density, the order of the $\mathrm{ABC}$ and the numerical stability.
\end{abstract}

Index terms-Finite element methods, time domain analysis, propagation.

I. INTRODUCTION

The numerical analysis of time domain wave equation problems is a well known technique. Yee [1] first developed a finite difference time domain (FDTD) algorithm which is now widely used to solve transient phenomena. With these FDTD methods it is however difficult to modelize complex geometries with curved surfaces because they use conventional rectangular grids. On the other hand the finite element (FE) method appears to be more powerful in handling complicated geometries with triangular meshes.

We have previously developed a $2 \mathrm{D}$ and $3 \mathrm{D}$ Finite Element formulation for unbounded microwave problems in the frequency domain [2]. This formulation is written directly in term of electric or magnetic field. The open boundary is modeled using an absorbing boundary condition (ABC). We show in this paper how this formulation can be extended in $2 \mathrm{D}$ in order to modelize time domain problems.

We present in a first part the absorbing boundary condition and the corner condition used to modelize the open boundary. The FE formulation and the numerical time scheme is then described. In a third part, the effect of numerical parameters such as the order and the angle of the $\mathrm{ABC}$, the mesh density and the time stepping is studied. Two examples of scattering by a plane wave are finally given.

II. HIGDON ABSORBING BOUNDARY CONDITION

Because the spatial domain is unbounded, an artificial boundary -with the corresponding $\mathrm{ABC}$ - has to be introduced

Manuscript received June 1, 1998.

L. Nicolas, laurent@trotek.ec-lyon.fr; C. Vollaire, vollair@trotek.eclyon.fr; A. Nicolas, nicolas@trotek.ec-lyon.fr; http://cegely.ec-lyon.fr/ to make the computational domain finite. Several types of $\mathrm{ABC}$ have been previously developed [3]. Because we consider a rectangular outer boundary, we have chosen the Higdon condition [4]:

$$
H_{m} h_{z}=\left(\prod_{j=1}^{m}\left(\cos \alpha_{j} \frac{\partial}{\partial t}-c \frac{\partial}{\partial x}\right)\right) h_{z}=0
$$

where $h_{z}$ is the 2D magnetic field and $c$ is the velocity of the light.

This boundary condition is perfectly absorbing for a plane wave hitting the boundary at one of the angles $\pm \alpha_{j}$. Note that, for $\alpha_{j}=0$, this $A B C$ is equivalent to that of Engquist-Majda [5]. At second order, (1) becomes:

$$
\begin{aligned}
& \frac{\partial^{2} h_{z}}{\partial x \partial t}+\frac{\alpha}{c} \frac{\partial^{2} h_{z}}{\partial t^{2}}+c \beta \frac{\partial^{2} h_{z}}{\partial y^{2}}=0 \\
& \text { with } \alpha=-\frac{1+\cos \alpha_{1} \cos \alpha_{2}}{\cos \alpha_{1}+\cos \alpha_{2}} \text { and } \beta=\frac{1}{\cos \alpha_{1}+\cos \alpha_{2}}
\end{aligned}
$$

As previously reported in several papers [5]-[6], because a rectangular computational domain is used, specific corner condition has to be introduced at the corners of the external boundary. This condition is obtained by combining the wave equation with the $\mathrm{ABC}$ written for each $\mathrm{x}$ and $\mathrm{y}$ axis intersecting at the corner. This allows to eliminate the second order spatial derivatives existing in the $\mathrm{ABC}$, and it leads to the corner condition:

$$
\begin{aligned}
& \frac{\partial \mathrm{h}_{\mathrm{z}}}{\partial \mathrm{x}}+\frac{\partial \mathrm{h}_{\mathrm{z}}}{\partial \mathrm{y}}-\frac{\gamma}{\mathrm{c}} \frac{\partial \mathrm{h}_{\mathrm{z}}}{\partial \mathrm{t}}=0 \\
& \quad \text { with } \gamma=\frac{2 \cos \alpha_{1} \cos \alpha_{2}+1}{\left(\cos \alpha_{1}+\cos \alpha_{2}\right)} \text { or } \gamma=\frac{3}{2} \text { for } \alpha_{1}=\alpha_{2}=0^{\circ}
\end{aligned}
$$

III. FinIte Element Time Domain SCHEME

The second order $\mathrm{ABC}$ is introduced by differentiating the time-varying wave equation with respect to the time [6]. After multiplication by a weighting function $w$ and integration in space, the $\mathrm{ABC}$ and the corner condition are inserted in the line integral of the Galerkin form. Written for the magnetic field $h_{z}$, the FE formulation is given by (4).

The time discretization is a four level scheme involving the times $k+1, k, k-1$ and $k-2$. It is centered at time $k-1 / 2$. The final FETD formulation is given by (5). 


$$
\begin{aligned}
& \mu \frac{\partial^{3}}{\partial \mathrm{t}^{3}} \int_{2} \mathrm{w} \cdot \mathrm{h}_{\mathrm{z}} \mathrm{d} \Omega+\frac{1}{\varepsilon} \frac{\partial}{\partial \mathrm{t}} \int_{2} \nabla w \cdot \nabla \mathrm{h}_{\mathrm{z}} \mathrm{d} \Omega
\end{aligned}
$$

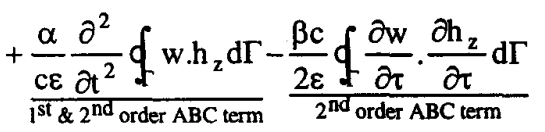

$$
\begin{aligned}
& +\left.\frac{\beta}{\gamma \varepsilon} \sum_{\text {corners }} w \cdot \frac{\partial h_{z}}{\partial t}\right|_{\text {comers }}=-\frac{\partial}{\partial t} \int_{h} w \cdot j \mathrm{~d} \Omega \\
& \left(\frac{\mu}{\Delta \mathrm{t}^{3}}[\mathbf{A}]+\frac{\alpha}{2 \varepsilon c \Delta \mathrm{t}^{2}}[\mathbf{C}]\right)_{\mathrm{z}}^{\mathrm{k}+1} \\
& +\left(-\frac{3 \mu}{\Delta \mathrm{t}^{3}}[\mathbf{A}]+\frac{1}{\varepsilon \Delta t}[\mathbf{B}]-\frac{\alpha}{2 \varepsilon c \Delta \mathrm{t}^{2}}[\mathbf{C}]-\frac{\beta \mathbf{c}}{2 \varepsilon}[\mathbf{D}]+\left.\frac{\beta \gamma}{\varepsilon \Delta t}\right|_{\text {corners }}\right) h_{z}^{k} \\
& +\left(\frac{3 \mu}{\Delta \mathrm{t}^{3}}[\mathbf{A}]-\frac{1}{\varepsilon \Delta \mathrm{t}}[\mathbf{B}]-\frac{\alpha}{2 \varepsilon c \Delta \mathrm{t}^{2}}[\mathbf{C}]-\frac{\beta \mathbf{c}}{2 \varepsilon}[\mathbf{D}]-\left.\frac{\beta \gamma}{\varepsilon \Delta t}\right|_{\text {comers }}\right) \mathbf{h}_{\mathbf{z}}^{\mathrm{k}-1} \\
& \left.+\left(-\frac{\mu}{\Delta \mathrm{t}^{3}}[\mathbf{A}]+\frac{\alpha}{2 \varepsilon \mathrm{c} \Delta \mathrm{t}^{2}}[\mathbf{C}]\right) \mathrm{h}_{\mathrm{z}}^{\mathrm{k}-2}=\frac{1}{\Delta \mathrm{t}}(\mathbf{J}]^{\mathrm{k}}-[\mathbf{J}]^{\mathrm{k}-1}\right) \\
& \text { with }[\mathbf{A}]=\int_{2} w \cdot h_{z} \mathrm{~d} \Omega,[\mathbf{B}]=\int_{2} \nabla w \cdot \nabla h_{2} \mathrm{~d} \Omega \\
& {[\mathbf{C}]=\oint w \cdot h_{z} \mathrm{~d} \Gamma,[\mathbf{D}]=\oint \frac{\partial \mathrm{w}}{\partial \tau} \cdot \frac{\partial \mathrm{h}_{\mathrm{z}}}{\partial \tau} \mathrm{d} \Gamma}
\end{aligned}
$$

Note that matrices $[\mathbf{A}],[\mathbf{B}],[\mathbf{C}]$ and $[\mathbf{D}]$ are the same than those appearing in the frequency domain formulation [2].

In (5), the mass matrix used to compute the values of $h_{z}$ at the step $k+1$ is not diagonal. The time scheme is implicit and a matrix inversion is required at each time step. In order to reduce the solution procedure to a vector-vector multiplication, the numerical time scheme is made explicit by row sum lumping [7]: the diagonal term of each row of the lumped matrix is the sum of all the terms in the row of the consistent matrix. The other terms of the line are canceled. This procedure is used for matrices $[A]$ and $[C]$ :

$$
A_{\mathrm{ii}}^{\text {lumped }}=\sum_{\mathrm{j}=1}^{\mathrm{n}} \mathrm{A}_{\mathrm{ij}} \text { and } \mathrm{A}_{\mathrm{ij}, \mathrm{i} \neq \mathrm{j}}^{\text {lumed }}=0
$$

This trick is possible for two reasons. First, first order triangles are used. With second order triangles, because the shape functions can be negative, diagonal terms can become equal to 0 . Second, [A] and [C] involve only the finite element basis functions, and not their derivatives.

\section{NUMERICAL EXPERIMENTS}

In this section, numerical experiments are performed in order to observe the effects of some parameters such as the corner condition, the order of the $A B C$, the mesh density and the time stepping.

\section{A. Test Problem}

It concerns a pulse in the free space [6]. The F.E. domain is a $0.5 \mathrm{~m}$ side square. The middle of the square is $(0 ., 0$.$) , the$ source is a circle of radius $0.04 \mathrm{~m}$, and the observation point $O^{\prime}$ is located close to one corner -at coordinates $(0.2,0.2)$ The source current is given by:

$$
\begin{gathered}
j(t)=10^{10} \cdot(1-r / 0.04) \cdot e^{-10\left(1-\frac{c t}{0.05}\right)^{2}} \\
\text { for } r \leq 0.04
\end{gathered}
$$

Default values for the different tests are:

- time step: $\Delta t=5$ ps

- mesh density: $6.75 \mathrm{~mm}$ ( 1 node every $6.75 \mathrm{~mm}$ )

- angles of $A B C: \alpha_{1}=\alpha_{2}=0^{\circ}$ (Engquist-Majda)

\section{B. Study of the order of the $A B C$}

A reference solution is obtained on a larger domain, so that no spurious reflection comes back from the boundaries during the considered computation time. Fig. 1 compares the magnetic field plotted at the observation point when using a $1^{\text {st }}$ order $A B C$, a $2^{\text {nd }}$ order $A B C$, with and without corner condition. Clearly, the $2^{\text {nd }}$ order $A B C$ has to be used, and the corner condition improves widely its accuracy. However, there is still some reflections coming back from the boundary (see the time steps between 175 and 200). On the other hand, the mass lumping does not seem to damage the results.

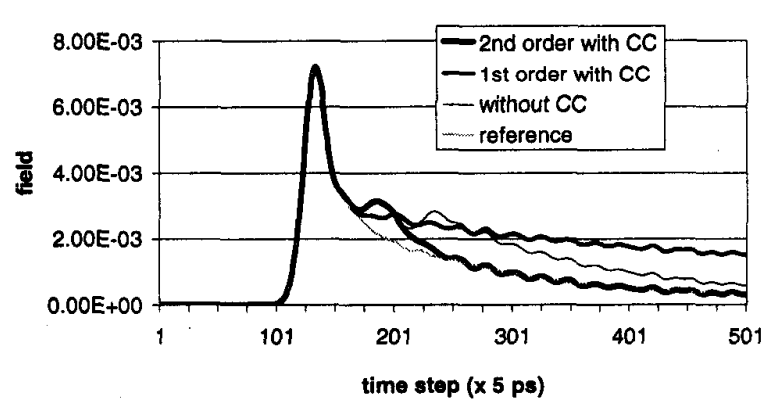

Fig. 1. Magnetic field near the comer of the F.E. domain. Comparison of different conditions (CC: comer condition)

\section{Study of the angle of the $A B C$}

A fine study of the main reflection occurring between the

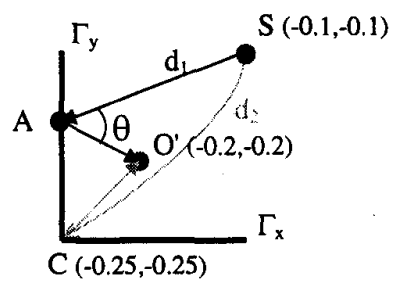

$$
\begin{aligned}
& \text { From } S \text { to } O^{\prime} \text { via } A: \\
& \begin{array}{l}
\mathrm{d}_{1}=0.223 \mathrm{~m} \\
\Delta \mathrm{t}_{1}=\mathrm{d}_{1} / \mathrm{c} \approx 150 \times 5 \mathrm{ps} \\
\Rightarrow \mathrm{t}_{1} \approx 190 \times 5 \mathrm{ps} \\
\text { From } S \text { to } \mathrm{O}^{\prime} \text { via } \mathrm{C}: \\
\mathrm{d}_{2}=0.283 \mathrm{~m} \\
\Delta \mathrm{t}_{2}=\mathrm{d}_{2} / \mathrm{c} \approx 190 \times 5 \mathrm{ps} \\
\Rightarrow \mathrm{t}_{2} \approx 230 \times 5 \mathrm{ps}
\end{array}
\end{aligned}
$$

Fig.2. Reflections coming back from the boundary and from the corner to the observation point $\mathrm{O}^{\prime}$. 
time steps 175 and 200 shows that the wave is coming back from the boundary itself and not from the corner:

Let $d_{1}$ be the distance between the source $S$ and the observation point $\mathrm{O}^{\prime}$, including the reflection at the point $\mathrm{A}$ of the boundary: $d_{1}=0.223 \mathrm{~m}$, and the corresponding time for the wave to propagate from $S$ to $O^{\prime}$ is $d t_{1}=750$ ps (Fig.2). Because the source is maximal in $S$ at the time 200 ps, this maximum will occur at $O^{\prime}$ at the time $T_{1}=950 \mathrm{ps}$, that is to say around the time step \#190 (Fig. 1 and Fig. 3).

Let $d_{2}$ be the distance between $S$ and $O^{\prime}$, including the reflection at the corner $\mathrm{C}$. The same reasoning leads to a maximal value of the field in $\mathrm{O}^{\prime}$ at the time step \#230. As shown in Fig.3, if no corner condition is used, 2 spurious reflections occur. On the other hand the reflection coming from the corner disappears when the corner condition is used.

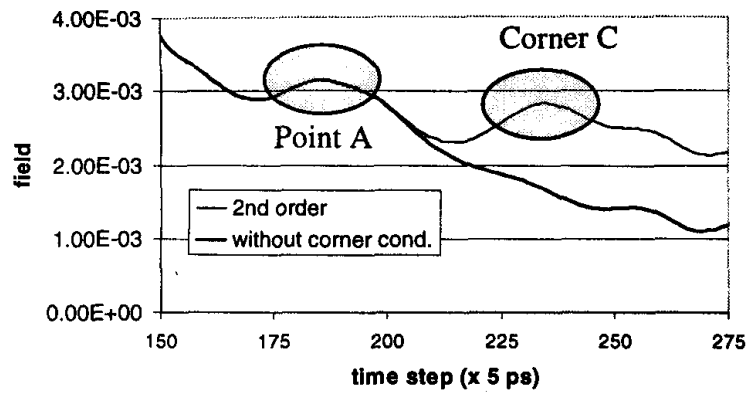

Fig. 3. Zoom of the Fig.1 between the time steps \#150 and \#275.

Fig. 4 shows the effect of several values of the angles $\alpha_{j}$ in the expression of the ABC (1). It seems that the main reflection may be more or less erased. However, in most applications, the direction in which the waves approach the boundary is unknown. Moreover, this direction may be different for each point of the boundary, so that no preferential angle can be determined.

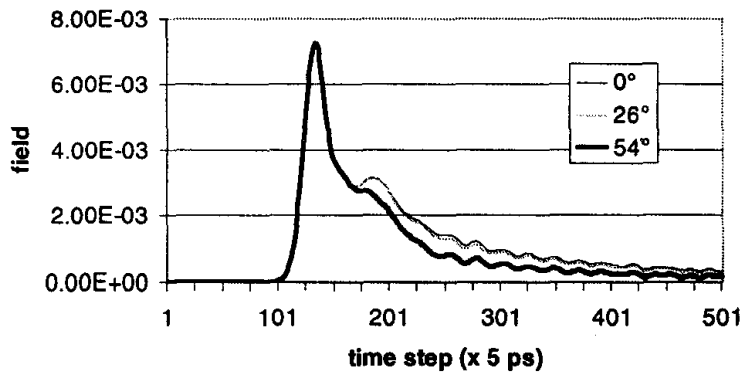

Fig.4. Magnetic field at $\mathrm{O}^{\prime}$ for various angles of the $\mathrm{ABC}$.

\section{Study of the mesh density}

The mesh is made of first order triangles. Several values of the mesh density have been tested, from 1 node every $10 \mathrm{~mm}$ to 1 node every $3.4 \mathrm{~mm}$ (Table I). Note that the solving time increases linearly with the number of nodes. Fig. 5 shows the time response at the point $\mathrm{O}^{\prime}$ for a $3.4 \mathrm{~mm}$ density mesh. Compared to Fig.1, the numerical oscillations have been removed. This is confirmed by Fig.6, where the relative difference at the point $O^{\prime}$ between the field computed with each mesh density and the field computed with the reference mesh density $(\mathrm{d}=3.4 \mathrm{~mm})$ is shown.

TABLE I

CPU TIMES FOR SEVERAL VALUES OF THE MESH DENSITY

\begin{tabular}{cccc}
\hline Nodes & Density $(\mathrm{mm})$ & Assembling time (s) & Solving per iteration (ms) \\
\hline 2737 & 10 & 14 & 32 \\
6549 & 6.75 & 63 & 73 \\
12494 & 4.1 & 214 & 138 \\
15813 & 3.4 & 339 & 174 \\
\hline
\end{tabular}

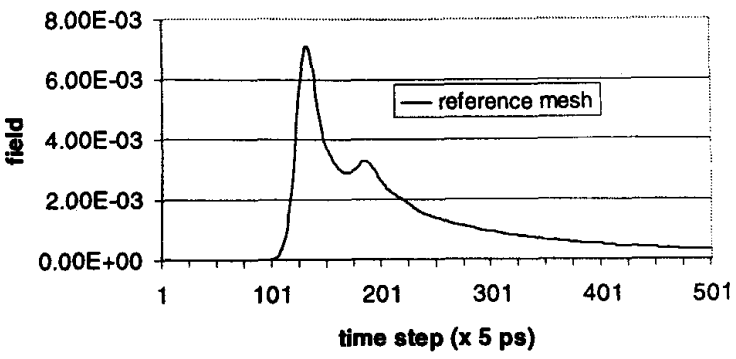

Fig. 5. Field at the point $O^{\prime}$ for the reference mesh (density: $3.4 \mathrm{~mm}$ ).

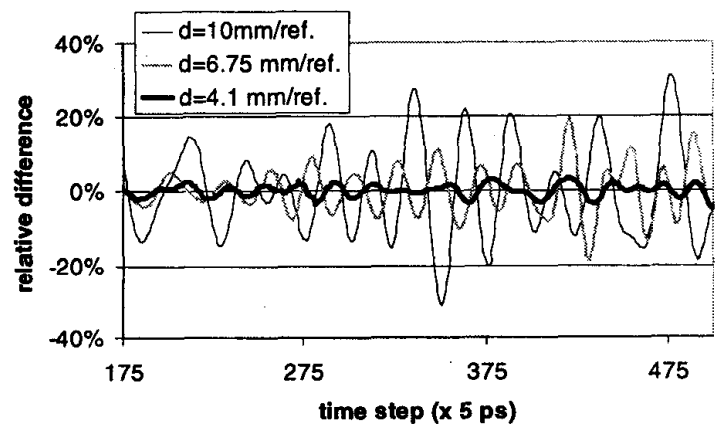

Fig. 6. Comparison at the point $O^{\prime}$ between the time response computed with several mesh densities. Reference value is obtained with 1 node $/ 3.4 \mathrm{~mm}$.

\section{E. Numerical stability}

The stability criterion of the time step is the same as previously reported in many papers. For triangular elements it may be written as [8]:

$$
c \Delta t \leq r_{c c}
$$

where $r_{c c}$ is the radius of the circumcircle of the triangular elements.

In the case of the $6.75 \mathrm{~mm}$ density mesh, the minimal distance between 2 adjacent nodes in the source region is about $3.2 \mathrm{~mm}$, leading to a maximal time step equal to $\Delta t=6$ ps. Different numerical tests have been performed for time steps varying from $6 \mathrm{ps}$ to $0.6 \mathrm{ps}$, and no numerical discrepancy has been observed. 


\section{COMPUTATION OF EXAMPLES}

A first example is the scattering by a perfect electric conducting cylinder when illuminated by a $3 \mathrm{GHz}$ TM plane wave. The upper part of Fig. 7 shows the frequency domain response while the lower part shows the time domain response when the steady state has been established. Both computations are performed with the same mesh, corresponding to 12.8 nodes per wavelength. Fig. 8 compares the magnetic field $\mathrm{Hz}$ along the cylinder boundary. Slight differences in the shadow region may be observed. Since $t$ same result is obtained with a larger domain, these differences are certainly due to numerical dispersion [9].

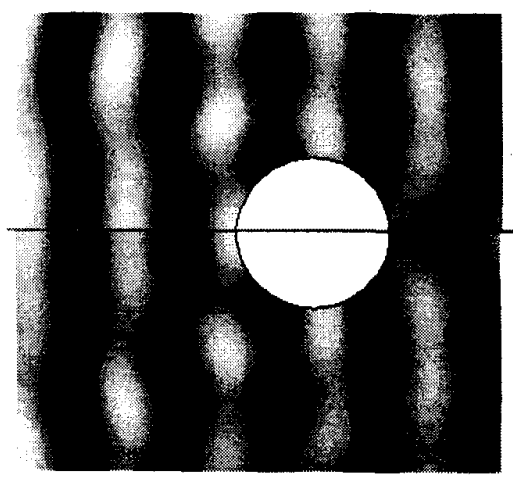

Frequency domain

TM mode, $3 \mathrm{GHz}$ CPU time:

assembling: $26 \mathrm{~s}$ solving: $12 \mathrm{~s}$

3834 nodes 1836 elements

Time stepping: $\Delta v=0.5 \mathrm{e}^{-11} \mathrm{~s}$ CPU time for 401 iterations: assembling: $24 \mathrm{~s}$ solving: $33 \mathrm{~s}$

Time domain steady state

Fig. 7. Scattering by a pec cylinder. Comparison between frequency domain and time domain computations when steady state is established.

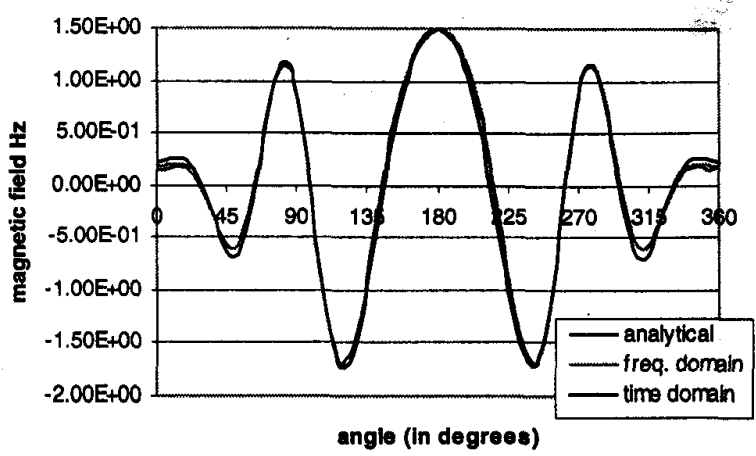

Fig. 8. Comparison of the magnetic field along the cylinder boundary between analytical, frequency domain and time domain solutions.

As large example, the illumination of an airplane by a TM plane wave is presented (Fig. 9). The frequency is 0.328 GHz. The mesh is constituted of 23718 nodes (density=9 nodes $(\lambda)$, and the time step is $\Delta t=0.1 \mathrm{~ns}$. Assembling time is $758 \mathrm{~s}$, while the solving time for 1501 iterations is $778 \mathrm{~s}$.

\section{CONCLUSION}

A 2D finite element time domain formulation for the modeling of electromagnetic wave propagation has been presented. The open boundary is modeled using an absorbing boundary condition improved by a corner condition. The time domain scheme is made explicit by mass lumping. Several numerical tests have shown the efficiency of this formulation.

REFERENCES

[1] K.S. Yee, "Numerical solution of initial boundary value problems involving Maxwell's equations in isotropic media," IEEE Trans. Antennas and Prop., vol. 14, pp. 302-307, 1966.

[2] J.L. Yao-Bi, L. Nicolas, A. Nicolas, "2D Electromagnetic Scattering by Simple Shapes: a Quantification of the Error due to Open Boundary," IEEE Trans. on Mag., vol. 29, n² 2, pp. 1830-1834, March 1993.

[3] D. Givoli, "Non-reflecting Boundary Conditions," Journal of Computational Physics, vol. 94, pp.1-29, 1991.

[4] R.L. Higdon, "Absorbing boundary conditions for difference approximations to the multi-dimensional wave equation," Math. Comput., vol. 47, $n^{\circ} 176$, pp.437-459, 1986.

[5] B. Engquist, A. Majda, "Radiation boundary conditions for acoustic and elastic wave calculations," Comm. Pure Appl. Math., vol. 32, pp. 313$357,1979$.

[6] A. Bamberger, P. Joly, J.E. Roberts, "Second-order absorbing boundary conditions for the wave equation: a solution for the comer problem," SIAM J. Numer. Anal., vol. 27, $\mathrm{n}^{\circ}$ 2, pp. 323-352, April 1990.

[7] O. Zienkiewicz, R. Taylor, The Finite Element Method, Mc Graw-Hill, 1991.

[8] K. Choi, S.J. Salon, K.A. Connor, L.F. Libelo, S.Y. Hahn, "Time domain finite element analysis of high power microwave aperture antennas," IEEE trans. on Mag., vol. 31, n³, pp. 1622-1625, 1995.

[9] A.C. Cangellaris, "Time-domain finite methods for electromagnetic wave propagation and scattering," IEEE Trans. on Mag., vol. 27, $\mathrm{n}^{\circ} 5$, pp.3780-3785, 1991.
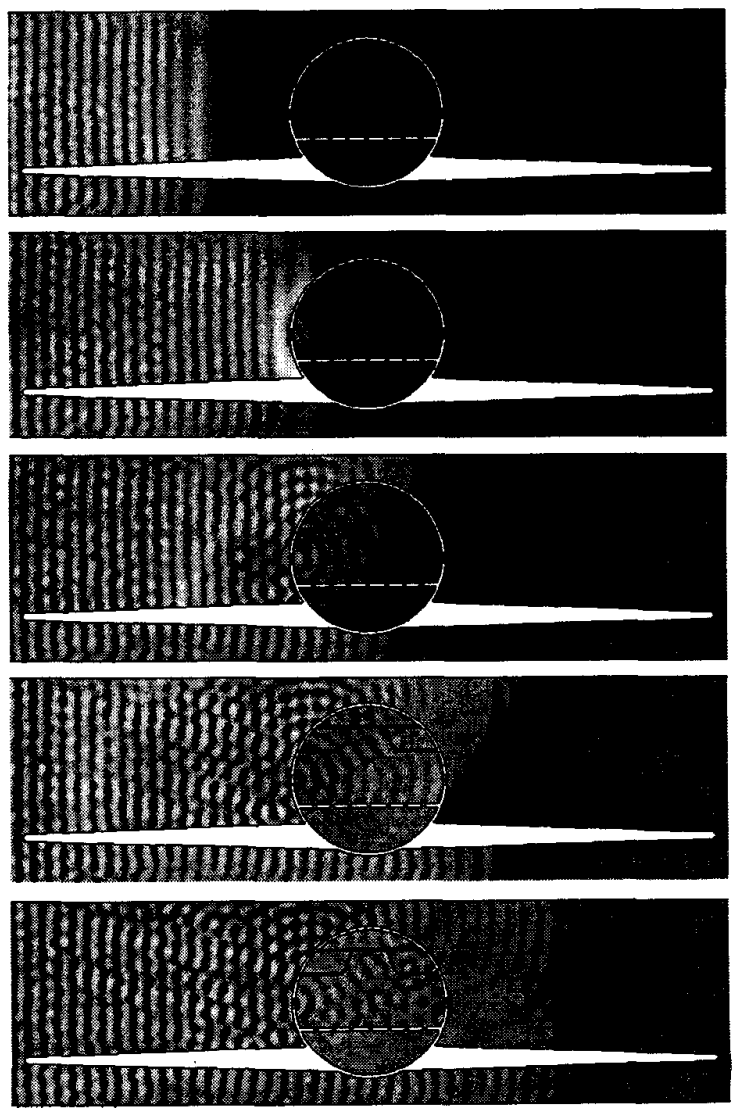

Fig. 9. Illumination of an airplane by a $0.328 \mathrm{GHz}$ plane wave. 